\title{
On the Deformations and Derivations of $n$-Ary Multiplicative Hom-Nambu-Lie Superalgebras
}

\author{
Baoling Guan, ${ }^{1}$ Liangyun Chen, ${ }^{2}$ and Yao $\mathrm{Ma}^{2}$ \\ ${ }^{1}$ College of Sciences, Qiqihar University, Qiqihar 161006, China \\ ${ }^{2}$ School of Mathematics and Statistics, Northeast Normal University, Changchun 130024, China \\ Correspondence should be addressed to Liangyun Chen; chenly640@nenu.edu.cn
}

Received 21 March 2014; Accepted 19 May 2014; Published 5 June 2014

Academic Editor: Yao-Zhong Zhang

Copyright (c) 2014 Baoling Guan et al. This is an open access article distributed under the Creative Commons Attribution License, which permits unrestricted use, distribution, and reproduction in any medium, provided the original work is properly cited.

\begin{abstract}
We introduce the relevant concepts of $n$-ary multiplicative Hom-Nambu-Lie superalgebras and construct three classes of $n$-ary multiplicative Hom-Nambu-Lie superalgebras. As a generalization of the notion of derivations for $n$-ary multiplicative HomNambu-Lie algebras, we discuss the derivations of $n$-ary multiplicative Hom-Nambu-Lie superalgebras. In addition, the theory of one parameter formal deformation of $n$-ary multiplicative Hom-Nambu-Lie superalgebras is developed by choosing a suitable cohomology.
\end{abstract}

\section{Introduction}

The notion of $n$-Lie algebras was introduced by Filippov in 1985 in [1]. The $n$-Lie algebra is a vector space endowed with an $n$-ary linear skew-symmetric product which satisfies the generalized Jacobi identity (also named Filippov identity). For $n=3$ this product is a special case of the Nambu bracket, introduced by Nambu in 1973 in [2], and was well known in physics, as a generalization of the Poisson bracket in Hamiltonian mechanics. $n$-Lie algebras are also useful in the research for M2-branes in the string theory and are closely linked to the Plücker relation in the literature in physics in [3-6].

In 1996, the concept of $n$-Lie superalgebras was firstly introduced by Daletskil and Kushnirevich in [7]. Moreover, Cantarini and Kac gave a more general concept of $n$-Lie superalgebras again in 2010 in [8]. $n$-Lie superalgebras are more general structures including $n$-Lie algebras, $n$-ary Nambu-Lie superalgebras, and Lie superalgebras.

The general Hom-algebra structures arose first in connection with quasi-deformation and discretizations of Lie algebras of vector fields. These quasi-deformations lead to quasiLie algebras, a generalized Lie algebra structure in which the skew-symmetry and Jacobi conditions are twisted. Hom-Lie algebras, Hom-associative algebras, Hom-Lie superalgebras,
Hom-bialgebras, $n$-ary Hom-Nambu-Lie algebras, and quasiHom-Lie algebras are discussed in [9-20]. Generalizations of $n$-ary algebras of Lie type and associative type by twisting the identities using linear maps have been introduced in [21].

The mathematical theory of deformations has proved to be a powerful tool in modeling physical reality. For example, (algebras associated with) classical quantum mechanics (and field theory) on a Poisson phase space can be deformed to (algebras associated with) quantum mechanics (and quantum field theory). The deformation of algebraic systems has been one of the problems that many mathematical researchers are interested in; Gerstenhaber studied the deformation theory of algebras in a series of papers [22-26]. For example, it has been extended to covariant functors from a small category to algebras. In $[27,28]$, it is, respectively, extended to algebra systems, bialgebras, Hopf algebras, Leibniz pairs, Poisson algebras, and so forth. In [23], Gerstenhaber developed a theory of deformation of associative and Lie algebras. His theory links cohomologies of these algebras and the Gerstenhaber bracket giving obstructions to deformations. Nijenhuis and Richardson noticed strong similarities between Gerstenhaber theory and the deformations of complex analytic structures on compact manifolds [29]. They axiomatized the theory of deformations via the introduction of graded Lie algebras [30]. One such example was given by the theory of deformations of 
homomorphisms [31]. Inspired by these works, we study the deformation theory of $n$-ary multiplicative Hom-Nambu-Lie superalgebras in this paper. In addition, the paper also discusses derivations of $n$-ary multiplicative Hom-Nambu-Lie superalgebras as a generalization of the notions of derivations for $n$-ary multiplicative Hom-Nambu-Lie algebras.

This paper is organized as follows. In Section 1, we introduce the relevant concepts of $n$-ary multiplicative HomNambu-Lie superalgebras and construct three classes of $n$-ary multiplicative Hom-Nambu-Lie superalgebras. In Section 2, the notion of derivation introduced for $n$-ary multiplicative Hom-Nambu-Lie algebras in [10] is extended to $n$-ary multiplicative Hom-Nambu-Lie superalgebras. In Section 3, the theory of deformations of $n$-ary multiplicative HomNambu-Lie superalgebras is developed by choosing a suitable cohomology.

Definition 1 (see [32]). An $n$-ary Nambu-Lie superalgebra is a pair $(\mathfrak{g},[\cdot, \ldots, \cdot])$ consisting of a $\mathbb{Z}_{2}$-graded vector space $\mathfrak{g}=$ $\mathfrak{g}_{\overline{0}} \oplus \mathfrak{g}_{\overline{1}}$ and a multilinear mapping $[\cdot, \ldots, \cdot]: \underbrace{\mathfrak{g} \times \cdots \times \mathfrak{g}}_{n} \rightarrow \mathfrak{g}$, satisfying

$$
\begin{aligned}
&\left|\left[x_{1}, \ldots, x_{n}\right]\right|=\left|x_{1}\right|+\cdots+\left|x_{n}\right|, \\
& {\left[x_{1}, \ldots, x_{i}, x_{i+1}, \ldots, x_{n}\right]=}-(-1)^{\left|x_{i}\right|\left|x_{i+1}\right|} \\
& \times\left[x_{1}, \ldots, x_{i+1}, x_{i}, \ldots, x_{n}\right], \\
& {\left[x_{1}, \ldots, x_{n-1},\left[y_{1}, \ldots, y_{n}\right]\right] } \\
&=\sum_{i=1}^{n}(-1)^{\left(\left|x_{1}\right|+\cdots+\left|x_{n-1}\right|\right)\left(\left|y_{1}\right|+\cdots+\left|y_{i-1}\right|\right)} \\
& \quad \cdot\left[y_{1}, \ldots, y_{i-1},\left[x_{1}, \ldots, x_{n-1}, y_{i}\right], y_{i+1}, \ldots, y_{n}\right],
\end{aligned}
$$

where $|x| \in \mathbb{Z}_{2}$ denotes the degree of a homogeneous element $x \in \mathfrak{g}$.

Definition 2. An $n$-ary Hom-Nambu-Lie superalgebra is a triple $(\mathfrak{g},[\cdot, \ldots, \cdot], \alpha)$ consisting of a $\mathbb{Z}_{2}$-graded vector space $\mathfrak{g}=\mathfrak{g}_{\overline{0}} \oplus \mathfrak{g}_{\overline{1}}$, a multilinear mapping $[\cdot, \ldots, \cdot]: \underbrace{\mathfrak{g} \times \cdots \times \mathfrak{g}}_{n} \rightarrow \mathfrak{g}$, and a family $\alpha=\left(\alpha_{i}\right)_{1 \leq i \leq n-1}$ of even linear maps $\alpha_{i}: \mathfrak{g} \rightarrow \mathfrak{g}$, satisfying

$$
\begin{gathered}
\left|\left[x_{1}, \ldots, x_{n}\right]\right|=\left|x_{1}\right|+\cdots+\left|x_{n}\right|, \\
{\left[x_{1}, \ldots, x_{i}, x_{i+1}, \ldots, x_{n}\right]=-(-1)^{\left|x_{i}\right|\left|x_{i+1}\right|}} \\
\times\left[x_{1}, \ldots, x_{i+1}, x_{i}, \ldots, x_{n}\right], \\
{\left[\alpha_{1}\left(x_{1}\right), \ldots, \alpha_{n-1}\left(x_{n-1}\right),\left[y_{1}, \ldots, y_{n}\right]\right]} \\
=\sum_{i=1}^{n}(-1)^{\left(\left|x_{1}\right|+\cdots+\left|x_{n-1}\right|\right)\left(\left|y_{1}\right|+\cdots+\left|y_{i-1}\right|\right)} \\
\cdot\left[\alpha_{1}\left(y_{1}\right), \ldots, \alpha_{i-1}\left(y_{i-1}\right),\left[x_{1}, \ldots, x_{n-1}, y_{i}\right],\right. \\
\left.\cdot \alpha_{i}\left(y_{i+1}\right), \ldots, \alpha_{n-1}\left(y_{n}\right)\right],
\end{gathered}
$$

where $|x| \in \mathbb{Z}_{2}$ denotes the degree of a homogeneous element $x \in \mathfrak{g}$.
An $n$-ary Hom-Nambu-Lie superalgebra $(\mathfrak{g},[\cdot, \ldots, \cdot], \alpha)$ is multiplicative, if $\alpha=\left(\alpha_{i}\right)_{1 \leq i \leq n-1}$ with $\alpha_{1}=\cdots=\alpha_{n-1}=\alpha$ and satisfying

$\alpha\left[x_{1}, \ldots, x_{n}\right]=\left[\alpha\left(x_{1}\right), \ldots, \alpha\left(x_{n}\right)\right], \quad \forall x_{1}, x_{2}, \ldots, x_{n} \in \mathfrak{g}$.

If the $n$-ary Hom-Nambu-Lie superalgebra $(\mathfrak{g},[\cdot, \ldots, \cdot], \alpha)$ is multiplicative, then (4) can be read as

$$
\begin{gathered}
{\left[\alpha\left(x_{1}\right), \ldots, \alpha\left(x_{n-1}\right),\left[y_{1}, \ldots, y_{n}\right]\right]} \\
=\sum_{i=1}^{n}(-1)^{\left(\left|x_{1}\right|+\cdots+\left|x_{n-1}\right|\right)\left(\left|y_{1}\right|+\cdots+\left|y_{i-1}\right|\right)} \\
\cdot\left[\alpha\left(y_{1}\right), \ldots, \alpha\left(y_{i-1}\right),\left[x_{1}, \ldots, x_{n-1}, y_{i}\right],\right. \\
\left.\alpha\left(y_{i+1}\right), \ldots, \alpha\left(y_{n}\right)\right] .
\end{gathered}
$$

It is clear that $n$-ary Hom-Nambu-Lie algebras and HomLie superalgebras are particular cases of $n$-ary Hom-NambuLie superalgebras. In the sequel, when the notation " $|x|$ " appears, it means that $x$ is a homogeneous element of degree $|x|$.

Definition 3. Let $(\mathfrak{g},[\cdot, \ldots, \cdot], \alpha)$ and $\left(\mathfrak{g}^{\prime},[\cdot, \ldots, \cdot]^{\prime}, \alpha^{\prime}\right)$ be two $n$-ary Hom-Nambu-Lie superalgebras, where $\alpha=\left(\alpha_{i}\right)_{1 \leq i \leq n-1}$ and $\alpha^{\prime}=\left(\alpha_{i}^{\prime}\right)_{1 \leq i \leq n-1}$. A linear map $f: \mathfrak{g} \rightarrow \mathfrak{g}$ is an $n$-ary Hom-Nambu-Lie superalgebra morphism if satisfies

$$
\begin{gathered}
f\left[x_{1}, \ldots, x_{n}\right]=\left[f\left(x_{1}\right), \ldots, f\left(x_{n}\right)\right]^{\prime}, \\
f \circ \alpha_{i}=\alpha_{i}^{\prime} \circ f, \quad \forall i=1, \ldots, n-1 .
\end{gathered}
$$

Theorem 4. Let $(\mathfrak{g},[\cdot, \ldots, \cdot], \alpha)$ be an $n$-ary multiplicative Hom-Nambu-Lie superalgebra and let $\beta: \mathfrak{g} \rightarrow \mathfrak{g}$ be a morphism of $g$ such that $\beta \circ \alpha=\alpha \circ \beta$. Then $(\mathfrak{g}, \beta \circ[\cdot, \ldots, \cdot], \beta \circ \alpha)$ is an $n$-ary multiplicative Hom-Nambu-Lie superalgebra.

Proof. Put $[\cdot, \ldots, \cdot]_{\beta}:=\beta \circ[\cdot, \ldots, \cdot]$. Then

$$
\begin{aligned}
(\beta \circ \alpha)\left[x_{1}, \ldots, x_{n}\right]_{\beta} & =(\beta \circ \alpha)\left(\beta\left[x_{1}, \ldots, x_{n}\right]\right) \\
& =\beta \circ(\alpha \circ \beta)\left[x_{1}, \ldots, x_{n}\right] \\
& =\beta \circ(\beta \circ \alpha)\left[x_{1}, \ldots, x_{n}\right] \\
& =\beta\left[\beta \circ \alpha\left(x_{1}\right), \ldots, \beta \circ \alpha\left(x_{n}\right)\right] \\
& =\left[\beta \circ \alpha\left(x_{1}\right), \ldots, \beta \circ \alpha\left(x_{n}\right)\right]_{\beta} ;
\end{aligned}
$$


that is, $\beta \circ \alpha$ is a morphism of $g$. Moreover, we have

$$
\begin{aligned}
& {\left[\beta \circ \alpha\left(x_{1}\right), \ldots, \beta \circ \alpha\left(x_{n-1}\right),\left[y_{1}, \ldots, y_{n}\right]_{\beta}\right]_{\beta}} \\
& =\beta\left[\beta \circ \alpha\left(x_{1}\right), \ldots, \beta \circ \alpha\left(x_{n-1}\right), \beta\left[y_{1}, \ldots, y_{n}\right]\right] \\
& =\beta^{2}\left[\alpha\left(x_{1}\right), \ldots, \alpha\left(x_{n-1}\right),\left[y_{1}, \ldots, y_{n}\right]\right] \\
& =\beta^{2}\left(\sum_{i=1}^{n}(-1)^{\left(\left|x_{1}\right|+\cdots+\left|x_{n-1}\right|\right)\left(\left|y_{1}\right|+\cdots+\left|y_{i-1}\right|\right)}\right. \\
& \left.\quad \times\left[\alpha\left(y_{1}\right), \ldots,\left[x_{1}, \ldots, x_{n-1}, y_{i}\right], \ldots, \alpha\left(y_{n}\right)\right]\right) \\
& =\sum_{i=1}^{n}(-1)^{\left(\left|x_{1}\right|+\cdots+\left|x_{n-1}\right|\right)\left(\left|y_{1}\right|+\cdots+\left|y_{i-1}\right|\right)} \\
& \quad \times \beta\left[\beta \circ \alpha\left(y_{1}\right), \ldots, \beta\left[x_{1}, \ldots, x_{n-1}, y_{i}\right], \ldots, \beta \circ \alpha\left(y_{n}\right)\right] \\
& =\sum_{i=1}^{n}(-1)^{\left(\left|x_{1}\right|+\cdots+\left|x_{n-1}\right|\right)\left(\left|y_{1}\right|+\cdots+\left|y_{i-1}\right|\right)} \\
& \quad \times\left[\beta \circ \alpha\left(y_{1}\right), \ldots,\left[x_{1}, \ldots, x_{n-1}, y_{i}\right]_{\beta}, \ldots, \beta \circ \alpha\left(y_{n}\right)\right]_{\beta} .
\end{aligned}
$$

Therefore, $(\mathfrak{g}, \beta \circ[\cdot, \ldots, \cdot], \beta \circ \alpha)$ is an $n$-ary multiplicative Hom-Nambu-Lie superalgebra.

In particular, we have the following example.

Example 5. Let $(\mathfrak{g},[\cdot, \ldots, \cdot])$ be an $n$-ary Nambu-Lie superalgebra and let $\rho: \mathfrak{g} \rightarrow \mathfrak{g}$ be an $n$-ary Nambu-Lie superalgebra endomorphism. Then $(\mathfrak{g}, \rho \circ[\cdot, \ldots, \cdot], \rho)$ is an $n$ ary multiplicative Hom-Nambu-Lie superalgebra.

Definition 6. Let $\left(\mathfrak{g},[\cdot, \ldots, \cdot]_{\mathfrak{g}}, \alpha\right)$ be an $n$-ary Hom-NambuLie superalgebra. A graded subspace $H \subseteq \mathfrak{g}$ is a Homsubalgebra of $\left(\mathfrak{g},[\cdot, \ldots, \cdot]_{\mathfrak{g}}, \alpha\right)$ if $\alpha(H) \subseteq H$ and $H$ is closed under the bracket operation $[\cdot, \ldots, \cdot]_{\mathfrak{g}}$; that is, $\left[u_{1}, \ldots, u_{n}\right]_{\mathfrak{g}} \in$ $H, \forall u_{1}, \ldots, u_{n} \in H$.

A graded subspace $H \subseteq \mathfrak{g}$ is a Hom-ideal of $\left(\mathfrak{g},[\cdot, \ldots, \cdot]_{\mathfrak{g}}\right.$, $\alpha)$ if $\alpha(H) \subseteq H$ and $\left[u_{1}, u_{2}, \ldots, u_{n}\right]_{\mathfrak{g}} \in H, \forall u_{1} \in H$, $u_{2}, \ldots, u_{n} \in \mathfrak{g}$.

Definition 7. Let $\left(\mathfrak{g}_{1},[\cdot, \ldots, \cdot]_{1}, \alpha\right)$ and $\left(\mathfrak{g}_{2},[\cdot, \ldots, \cdot]_{2}, \beta\right)$ be two $n$-ary multiplicative Hom-Nambu-Lie superalgebras. Suppose that $\phi: \mathfrak{g}_{1} \rightarrow \mathfrak{g}_{2}$ is a linear map. $\mathfrak{G}_{\phi}=\{(x, \phi(x)) \mid$ $\left.x \in \mathfrak{g}_{1}\right\} \subseteq \mathfrak{g}_{1} \oplus \mathfrak{g}_{2}$ is called as the graph of a linear map $\phi: \mathfrak{g}_{1} \rightarrow \mathfrak{g}_{2}$

Proposition 8. Given two n-ary multiplicative Hom-NambuLie superalgebras $\left(\mathfrak{g}_{1},[\cdot, \ldots, \cdot]_{\mathfrak{g}_{1}}, \alpha\right)$ and $\left(\mathfrak{g}_{2},[\cdot, \ldots, \cdot]_{\mathfrak{g}_{2}}, \beta\right)$, there is an n-ary multiplicative Hom-Nambu-Lie superalgebra $\left(\mathfrak{g}_{1} \oplus \mathfrak{g}_{2},[\cdot, \ldots, \cdot]_{\mathfrak{g}_{1} \oplus \mathfrak{g}_{2}}, \alpha+\beta\right)$, where the bilinear map $[\cdot, \ldots, \cdot]_{\mathfrak{g}_{1} \oplus \mathfrak{g}_{2}}: \wedge^{2}\left(\mathfrak{g}_{1} \oplus \mathfrak{g}_{2}\right) \rightarrow \mathfrak{g}_{1} \oplus \mathfrak{g}_{2}$ is given by

$$
\begin{array}{r}
{\left[u_{1}+v_{1}, \ldots, u_{n}+v_{n}\right]_{\mathfrak{g}_{1} \oplus \mathfrak{g}_{2}}=\left[u_{1}, \ldots, u_{n}\right]_{\mathfrak{g}_{1}}+\left[v_{1}, \ldots, v_{n}\right]_{\mathfrak{g}_{2}},} \\
\forall u_{i} \in \mathfrak{g}_{1}, v_{i} \in \mathfrak{g}_{2}(i=1,2, \ldots, n),
\end{array}
$$

and the linear map $(\alpha+\beta): \mathfrak{g}_{1} \oplus \mathfrak{g}_{2} \rightarrow \mathfrak{g}_{1} \oplus \mathfrak{g}_{2}$ is given by

$$
(\alpha+\beta)(u+v)=\alpha(u)+\beta(v), \quad \forall u \in \mathfrak{g}_{1}, \quad v \in \mathfrak{g}_{2} .
$$

Proof. For any $u_{i} \in \mathfrak{g}_{1}, v_{i} \in \mathfrak{g}_{2}$, we have

$$
\begin{aligned}
{[} & \left.u_{1}+v_{1}, \ldots, u_{i}+v_{i}, u_{i+1}+v_{i+1}, \ldots, u_{n}+v_{n}\right]_{\mathfrak{g}_{1} \oplus \mathfrak{g}_{2}} \\
= & {\left[u_{1}, \ldots, u_{i}, u_{i+1}, \ldots, u_{n}\right]_{\mathfrak{g}_{1}}+\left[v_{1}, \ldots, v_{i}, v_{i+1}, \ldots, v_{n}\right]_{\mathfrak{g}_{2}} } \\
= & -(-1)^{\left|u_{i}\right|\left|u_{i+1}\right|}\left[u_{1}, \ldots, u_{i+1}, u_{i}, \ldots, u_{n}\right]_{\mathfrak{g}_{1}} \\
& -(-1)^{\left|v_{i}\right|\left|v_{i+1}\right|}\left[v_{1}, \ldots, v_{i+1}, v_{i}, \ldots, v_{n}\right]_{\mathfrak{g}_{2}} \\
= & -(-1)^{\left|u_{i}\right|\left|u_{i+1}\right|} \\
& \times\left(\left[u_{1}, \ldots, u_{i}, u_{i+1}, \ldots, u_{n}\right]_{\mathfrak{g}_{1}}+\left[v_{1}, \ldots, v_{i}, v_{i+1}, \ldots, v_{n}\right]_{\mathfrak{g}_{2}}\right) \\
= & -(-1)^{\left|u_{i}\right|\left|u_{i+1}\right|} \\
& \times\left[u_{1}+v_{1}, \ldots, u_{i+1}+v_{i+1}, u_{i}+v_{i}, \ldots, u_{n}+v_{n}\right]_{\mathfrak{g}_{1} \oplus \mathfrak{g}_{2}} .
\end{aligned}
$$

The bracket is obviously supersymmetric. By a direct computation we have

$$
\begin{aligned}
& {\left[(\alpha+\beta)\left(u_{1}+v_{1}\right), \ldots,(\alpha+\beta)\left(u_{n-1}+v_{n-1}\right),\right.} \\
& \left.\left[x_{1}+y_{1}, \ldots, x_{n}+y_{n}\right]_{\mathfrak{g}_{1} \oplus \mathfrak{g}_{2}}\right]_{\mathfrak{g}_{1} \oplus \mathfrak{g}_{2}} \\
& =\left[\alpha\left(u_{1}\right)+\beta\left(v_{1}\right), \ldots, \alpha\left(u_{n-1}\right)+\beta\left(v_{n-1}\right)\right. \text {, } \\
& \left.\left[x_{1}+y_{1}, \ldots, x_{n}+y_{n}\right]_{\mathfrak{g}_{1} \oplus \mathfrak{g}_{2}}\right]_{\mathfrak{g}_{1} \oplus \mathfrak{g}_{2}} \\
& =\left[\alpha\left(u_{1}\right), \ldots, \alpha\left(u_{n-1}\right),\left[x_{1}, \ldots, x_{n}\right]_{\mathfrak{g}_{1}}\right]_{\mathfrak{g}_{1}} \\
& +\left[\beta\left(v_{1}\right), \ldots, \beta\left(v_{n-1}\right),\left[y_{1}, \ldots, y_{n}\right]_{\mathfrak{g}_{2}}\right]_{\mathfrak{g}_{2}} \\
& =\sum_{i=1}^{n}(-1)^{\left(\left|u_{1}\right|+\cdots+\left|u_{n-1}\right|\right)\left(\left|x_{1}\right|+\cdots+\left|x_{i-1}\right|\right)} \\
& \times\left(\left[\alpha\left(x_{1}\right), \ldots, \alpha\left(x_{i-1}\right),\left[u_{1}, \ldots, u_{n-1}, x_{i}\right]_{\mathfrak{g}_{1}},\right.\right. \\
& \left.\alpha\left(x_{i+1}\right), \ldots, \alpha\left(x_{n}\right)\right]_{\mathfrak{g}_{1}} \\
& +\left[\beta\left(y_{1}\right), \ldots, \beta\left(y_{i-1}\right),\left[v_{1}, \ldots, v_{n-1}, y_{i}\right]_{\mathfrak{g}_{2}},\right. \\
& \left.\left.\beta\left(y_{i+1}\right), \ldots, \beta\left(y_{n}\right)\right]_{\mathfrak{g}_{2}}\right) \\
& =\sum_{i=1}^{n}(-1)^{\left(\left|u_{1}\right|+\cdots+\left|u_{n-1}\right|\right)\left(\left|x_{1}\right|+\cdots+\left|x_{i-1}\right|\right)} \\
& \times\left[\alpha\left(x_{1}\right)+\beta\left(y_{1}\right), \ldots, \alpha\left(x_{i-1}\right)+\beta\left(y_{i-1}\right),\right. \\
& {\left[u_{1}, \ldots, u_{n-1}, x_{i}\right]_{\mathfrak{g}_{1}}+\left[v_{1}, \ldots, v_{n-1}, y_{i}\right]_{\mathfrak{g}_{2}},}
\end{aligned}
$$




$$
\begin{gathered}
\left.\alpha\left(x_{i+1}\right)+\beta\left(y_{i+1}\right), \ldots, \alpha\left(x_{n}\right)+\beta\left(y_{n}\right)\right]_{\mathfrak{g}_{1} \oplus \mathfrak{g}_{2}} \\
=\sum_{i=1}^{n}(-1)^{\left(\left|u_{1}\right|+\cdots+\left|u_{n-1}\right|\right)\left(\left|x_{1}\right|+\cdots+\left|x_{i-1}\right|\right)} \\
\times\left[(\alpha+\beta)\left(x_{1}+y_{1}\right), \ldots,(\alpha+\beta)\left(x_{i-1}+y_{i-1}\right),\right. \\
{\left[u_{1}+v_{1}, \ldots, u_{n-1}+v_{n-1}, x_{i}+y_{i}\right]_{\mathfrak{g}_{1} \oplus \mathfrak{g}_{2}},} \\
\left.(\alpha+\beta)\left(x_{i+1}+y_{i+1}\right), \ldots,(\alpha+\beta)\left(x_{n}+y_{n}\right)\right]_{\mathfrak{g}_{1} \oplus \mathfrak{g}_{2}} \\
=\sum_{i=1}^{n}(-1)^{\left(\left|u_{1}\right|+\cdots+\left|u_{n-1}\right|\right)\left(\left|x_{1}\right|+\cdots+\left|x_{i-1}\right|\right)} \\
\times\left[(\alpha+\beta)\left(x_{1}+y_{1}\right), \ldots,(\alpha+\beta)\left(x_{i-1}+y_{i-1}\right),\right. \\
{\left[u_{1}+v_{1}, \ldots, u_{n-1}+v_{n-1}, x_{i}+y_{i}\right]_{\mathfrak{g}_{1} \oplus \mathfrak{g}_{2}},} \\
\left.(\alpha+\beta)\left(x_{i+1}+y_{i+1}\right), \ldots,(\alpha+\beta)\left(x_{n}+y_{n}\right)\right]_{\mathfrak{g}_{1} \oplus \mathfrak{g}_{2}}
\end{gathered}
$$

The result follows.

Proposition 9. A linear map $\phi:\left(\mathfrak{g}_{1},[\cdot, \ldots, \cdot]_{\mathfrak{g}_{1}}, \alpha\right) \rightarrow$ $\left(\mathfrak{g}_{2},[\cdot, \ldots, \cdot]_{\mathfrak{g}_{2}}, \beta\right)$ is a morphism of $n$-ary multiplicative HomNambu-Lie superalgebras if and only if the graph $\mathfrak{W}_{\phi} \subseteq \mathfrak{g}_{1} \oplus \mathfrak{g}_{2}$ is a Hom-subalgebra of $\left(\mathfrak{g}_{1} \oplus \mathfrak{g}_{2},[\cdot, \ldots, \cdot]_{\mathfrak{g}_{1} \oplus \mathfrak{g}_{2}}, \alpha+\beta\right)$.

Proof. Let $\phi:\left(\mathfrak{g}_{1},[\cdot, \ldots, \cdot]_{\mathfrak{g}_{1}}, \alpha\right) \rightarrow\left(\mathfrak{g}_{2},[\cdot, \ldots, \cdot]_{\mathfrak{g}_{2}}, \beta\right)$ be a morphism of $n$-ary multiplicative Hom-Nambu-Lie superalgebras. Then

$$
\begin{aligned}
{\left[u_{1}+\right.} & \left.\phi\left(u_{1}\right), \ldots, u_{n}+\phi\left(u_{n}\right)\right]_{\mathfrak{g}_{1} \oplus \mathfrak{g}_{2}} \\
& =\left[u_{1}, \ldots, u_{n}\right]_{\mathfrak{g}_{1}}+\left[\phi\left(u_{1}\right), \ldots, \phi\left(u_{n}\right)\right]_{\mathfrak{g}_{2}} \\
& =\left[u_{1}, \ldots, u_{n}\right]_{\mathfrak{g}_{1}}+\phi\left[u_{1}, \ldots, u_{n}\right]_{\mathfrak{g}_{2}} .
\end{aligned}
$$

Then the graph $\mathfrak{G}_{\phi}$ is closed under the bracket operation $[\cdot, \ldots, \cdot]_{\mathfrak{g}_{1} \oplus \mathfrak{g}_{2}}$. Furthermore, we obtain

$$
(\alpha+\beta)(u+\phi(u))=\alpha(u)+\beta \circ \phi(u)=\alpha(u)+\phi \circ \alpha(u),
$$

which implies that $(\alpha+\beta)\left(\mathfrak{G}_{\phi}\right) \subseteq \mathfrak{G}_{\phi}$. Thus, $\mathfrak{G}_{\phi}$ is a Homsubalgebra of $\left(\mathfrak{g}_{1} \oplus \mathfrak{g}_{2},[\cdot, \ldots, \cdot]_{\mathfrak{g}_{1} \oplus \mathfrak{g}_{2}}, \alpha+\beta\right)$.

Conversely, if the graph $\mathfrak{G}_{\phi} \subseteq \mathfrak{g}_{1} \oplus \mathfrak{g}_{2}$ is a Hom-subalgebra of $\left(\mathfrak{g}_{1} \oplus \mathfrak{g}_{2},[\cdot, \ldots, \cdot]_{\mathfrak{g}_{1} \oplus \mathfrak{g}_{2}}, \alpha+\beta\right)$, then we have

$$
\begin{aligned}
& {\left[u_{1}+\phi\left(u_{1}\right), \ldots, u_{n}+\phi\left(u_{n}\right)\right]_{\mathfrak{g}_{1} \oplus \mathfrak{g}_{2}}=} {\left[u_{1}, \ldots, u_{n}\right]_{\mathfrak{g}_{1}} } \\
&+\phi\left[u_{1}, \ldots, u_{n}\right]_{\mathfrak{g}_{2}},
\end{aligned}
$$

which implies that

$$
\phi\left[u_{1}, \ldots, u_{n}\right]_{\mathfrak{g}_{2}}=\phi\left[u_{1}, \ldots, u_{n}\right]_{\mathfrak{g}_{1}} .
$$

Furthermore, $(\alpha+\beta)\left(\mathfrak{G}_{\phi}\right) \subset \mathfrak{G}_{\phi}$ yields that

$$
(\alpha+\beta)(u+\phi(u))=\alpha(u)+\beta \circ \phi(u) \in \mathfrak{G}_{\phi},
$$

which is equivalent to the condition $\beta \circ \phi(u)=\phi \circ \alpha(u)$; that is, $\beta \circ \phi=\phi \circ \alpha$. Therefore, $\phi$ is a morphism of $n$-ary multiplicative Hom-Nambu-Lie superalgebras.

\section{Derivations of $n$-Ary Multiplicative Hom- Nambu-Lie Superalgebras}

Let $(\mathfrak{g},[\cdot, \ldots, \cdot], \alpha)$ be an $n$-ary multiplicative Hom-NambuLie superalgebra. We denote by $\alpha^{k}$ the $k$-times compositions of $\alpha$. In particular, we set $\alpha^{0}=\mathrm{id}$.

Definition 10. For $k \geq 0$, we call $D \in \operatorname{End}(\mathfrak{g})$ an $\alpha^{k}$-derivation of the $n$-ary multiplicative Hom-Nambu-Lie superalgebra $(\mathfrak{g},[\cdot, \ldots, \cdot], \alpha)$ if

$$
D \circ \alpha=\alpha \circ D
$$

and for $x_{i} \in \mathfrak{g}(i=1, \ldots, n)$,

$$
\begin{aligned}
& D\left[x_{1}, \ldots, x_{n}\right] \\
& =\sum_{i=1}^{n}(-1)^{|D|\left(\left|x_{1}\right|+\cdots+\left|x_{i-1}\right|\right)} \\
& \quad \times\left[\alpha^{k}\left(x_{1}\right), \ldots, \alpha^{k}\left(x_{i-1}\right), D\left(x_{i}\right), \alpha^{k}\left(x_{i+1}\right), \ldots,\right. \\
& \left.\quad \alpha^{k}\left(x_{n}\right)\right] .
\end{aligned}
$$

We denote by $\operatorname{Der}_{\alpha^{k}}(\mathfrak{g})$ the set of $\alpha^{k}$-derivations of the $n$-ary multiplicative Hom-Nambu-Lie superalgebra $(\mathfrak{g},[\cdot, \ldots, \cdot], \alpha)$. Notice that we obtain classical derivations for $k=0$.

For $\mathscr{X} \in \mathfrak{g}^{\wedge^{n-1}}$ satisfying $\alpha(\mathscr{X})=\mathscr{X}$ and $k \geq 0$, we define the $\operatorname{map}_{k} \operatorname{ad}_{k}(\mathscr{X}) \in \operatorname{End}(\mathfrak{g})$ by

$$
\operatorname{ad}_{k}(\mathscr{X})(y)=\left[x_{1}, \ldots, x_{n-1}, \alpha^{k}(y)\right], \quad \forall y \in \mathfrak{g} .
$$

Then one has the following.

Lemma 11. The map $\operatorname{ad}_{k}(\mathscr{X})$ is an $\alpha^{k+1}$-derivation and is called an inner $\alpha^{k+1}$-derivation.

We denote by $\operatorname{Inn}_{\alpha^{k}}(\mathfrak{g})$ the $\mathbb{K}$-vector space generated by all inner $\alpha^{k+1}$-derivations. For any $D \in \operatorname{Der}_{\alpha^{k}}(\mathfrak{g})$ and $D^{\prime} \epsilon$ $\operatorname{Der}_{\alpha^{k^{\prime}}}(\mathfrak{g})$, we define their commutator $\left[D, D^{\prime}\right]=D \circ D^{\prime}-$ $(-1)^{|D|\left|D^{\prime}\right|} D^{\prime} \circ D$. Set $\operatorname{Der}(\mathfrak{g})=\oplus_{k \geq 0} \operatorname{Der}_{\alpha^{k}}(\mathfrak{g})$ and $\operatorname{Inn}(\mathfrak{g})=$ $\oplus_{k \geq 0} \operatorname{Inn}_{\alpha^{k}}(\mathfrak{g})$.

Lemma 12. For any $D \in \operatorname{Der}_{\alpha^{k}}(\mathfrak{g})$ and $D^{\prime} \in \operatorname{Der}_{\alpha^{k^{\prime}}}(\mathfrak{g})$, one has $\left[D, D^{\prime}\right] \in \operatorname{Der}_{\alpha^{k+k^{\prime}}}(\mathfrak{g})$. 
Proof. Let $x_{i} \in \mathfrak{g}, 1 \leq i \leq n . D \in \operatorname{Der}_{\alpha^{k}}(\mathfrak{g})$ and $D^{\prime} \in$ $\operatorname{Der}_{\alpha^{k^{k}}}(\mathfrak{g})$, and then

$$
\begin{aligned}
& D \circ D^{\prime}\left(\left[x_{1}, \ldots, x_{n}\right]\right) \\
& =D\left(\sum_{i=1}^{n}(-1)^{\left|D^{\prime}\right|\left(\left|x_{1}\right|+\cdots+\left|x_{i-1}\right|\right)}\right. \\
& \quad \times\left[\alpha^{k^{\prime}}\left(x_{1}\right), \ldots, \alpha^{k^{\prime}}\left(x_{i-1}\right),\right. \\
& \left.\left.D^{\prime}\left(x_{i}\right), \alpha^{k^{\prime}}\left(x_{i+1}\right), \ldots, \alpha^{k^{\prime}}\left(x_{n}\right)\right]\right) \\
& =\sum_{i=1}^{n}(-1)^{\left|D^{\prime}\right|\left(\left|x_{1}\right|+\cdots+\left|x_{i-1}\right|\right)} D \\
& \quad \times\left[\alpha^{k^{\prime}}\left(x_{1}\right), \ldots, \alpha^{k^{\prime}}\left(x_{i-1}\right),\right. \\
& \left.D^{\prime}\left(x_{i}\right), \alpha^{k^{\prime}}\left(x_{i+1}\right), \ldots, \alpha^{k^{\prime}}\left(x_{n}\right)\right] \\
& =\sum_{i=1}^{n}(-1)^{\left|D^{\prime}\right|\left(\left|x_{1}\right|+\cdots+\left|x_{i-1}\right|\right)}(-1)^{|D|\left(\left|x_{1}\right|+\cdots+\left|x_{i-1}\right|\right)}
\end{aligned}
$$

$$
\begin{gathered}
\cdot\left[\alpha^{k+k^{\prime}}\left(x_{1}\right), \ldots, \alpha^{k+k^{\prime}}\left(x_{i-1}\right), D \circ D^{\prime}\left(x_{i}\right),\right. \\
\left.\alpha^{k+k^{\prime}}\left(x_{i+1}\right), \ldots, \alpha^{k+k^{\prime}}\left(x_{n}\right)\right] \\
+\sum_{i<j}(-1)^{\left|D^{\prime}\right|\left(\left|x_{1}\right|+\cdots+\left|x_{i-1}\right|\right)}(-1)^{|D|\left(\left|x_{1}\right|+\cdots+\left|x_{j-1}\right|+\left|D^{\prime}\right|\right)} \\
\cdot\left[\alpha^{k+k^{\prime}}\left(x_{1}\right), \ldots, \alpha^{k}\left(D^{\prime}\left(x_{i}\right)\right), \ldots,\right. \\
+\sum_{i>j}(-1)^{\left|D^{\prime}\right| \mid\left(x_{1}|+\cdots+| x_{i-1} \mid\right)}(-1)^{|D|\left(\left|x_{1}\right|+\cdots+\left|x_{j-1}\right|\right)} \\
\cdot\left[\alpha^{k+k^{\prime}}\left(x_{1}\right), \ldots, \alpha^{k^{\prime}}\left(D\left(x_{j}\right)\right), \ldots,\right. \\
\left.\alpha^{k}\left(D^{\prime}\left(x_{i}\right)\right), \ldots, \alpha^{k+k^{\prime}}\left(x_{n}\right)\right], \\
=(-1)^{|D|\left|D^{\prime}\right|} D^{\prime} \circ D\left(\left[x_{1}, \ldots, x_{n}\right]\right) \\
=-(-1)^{\left|D \| D^{\prime}\right|} D^{\prime} \\
\times\left(\sum_{i=1}^{n}(-1)^{|D|\left(\left|x_{1}\right|+\cdots+\left|x_{i-1}\right|\right)}\right. \\
\left.\times\left[\alpha^{k}\left(x_{1}\right), \ldots, D\left(x_{i}\right), \ldots, \alpha^{k}\left(x_{n}\right)\right]\right) \\
\times-1)^{\left|D \| D^{\prime}\right|} \sum_{i=1}^{n}(-1)^{|D|\left(\left|x_{1}\right|+\cdots+\left|x_{i-1}\right|\right)} \\
\times D^{\prime}\left[\alpha^{k}\left(x_{1}\right), \ldots, D\left(x_{i}\right), \ldots, \alpha^{k}\left(x_{n}\right)\right]
\end{gathered}
$$

$$
\begin{aligned}
& =-(-1)^{|D \|| D^{\prime} \mid} \sum_{i=1}^{n}(-1)^{|D|\left(\left|x_{1}\right|+\cdots+\left|x_{i-1}\right|\right)}(-1)^{\left.\left|D^{\prime}\right||| x_{1}|+\cdots+| x_{i-1} \mid\right)} \\
& \cdot\left[\alpha^{k^{\prime}+k}\left(x_{1}\right), \ldots, \alpha^{k^{\prime}+k}\left(x_{i-1}\right), D^{\prime} \circ D\left(x_{i}\right),\right. \\
& \left.\alpha^{k^{\prime}+k}\left(x_{i+1}\right), \ldots, \alpha^{k^{\prime}+k}\left(x_{n}\right)\right] \\
& -(-1)^{|D \|| D^{\prime} \mid} \sum_{i<j}(-1)^{|D|\left(\left|x_{1}\right|+\cdots+\left|x_{i-1}\right|\right)}(-1)^{\left.\left|D^{\prime}\right||| x_{1}|+\cdots+| x_{j-1}|+| D \mid\right)} \\
& \cdot\left[\alpha^{k^{\prime}+k}\left(x_{1}\right), \ldots, \alpha^{k^{\prime}}\left(D\left(x_{i}\right)\right), \ldots,\right. \\
& \left.\alpha^{k}\left(D^{\prime}\left(x_{j}\right)\right), \ldots, \alpha^{k^{\prime}+k}\left(x_{n}\right)\right] \\
& -(-1)^{|D|\left|D^{\prime}\right|} \sum_{i>j}(-1)^{|D|\left(\left|x_{1}\right|+\cdots+\left|x_{i-1}\right|\right)}(-1)^{\left|D^{\prime}\right|\left(\left|x_{1}\right|+\cdots+\left|x_{j-1}\right|\right)} \\
& \cdot\left[\alpha^{k^{\prime}+k}\left(x_{1}\right), \ldots, \alpha^{k}\left(D^{\prime}\left(x_{j}\right)\right), \ldots,\right. \\
& \left.\alpha^{k^{\prime}}\left(D\left(x_{i}\right)\right), \ldots, \alpha^{k^{\prime}+k}\left(x_{n}\right)\right] \text {. }
\end{aligned}
$$

Then we obtain

$$
\begin{aligned}
& {\left[D, D^{\prime}\right]\left(\left[x_{1}, \ldots, x_{n}\right]\right)} \\
& =\left(D \circ D^{\prime}-(-1)^{|D|\left|D^{\prime}\right|} D^{\prime} \circ D\right)\left(\left[x_{1}, \ldots, x_{n}\right]\right) \\
& =\sum_{i=1}^{n}(-1)^{\left|D^{\prime}\right|\left(\left|x_{1}\right|+\cdots+\left|x_{i-1}\right|\right)}(-1)^{|D|\left(\left|x_{1}\right|+\cdots+\left|x_{i-1}\right|\right)} \\
& \times\left[\alpha^{k+k^{\prime}}\left(x_{1}\right), \ldots, \alpha^{k+k^{\prime}}\left(x_{i-1}\right),\right. \\
& \quad\left(D \circ D^{\prime}-(-1)^{|D|\left|D^{\prime}\right|} D^{\prime} \circ D\right)\left(x_{i}\right), \\
& =\sum_{i=1}^{n}(-1)^{\left(\left|D^{\prime}\right|+|D|\right)\left(\left|x_{1}\right|+\cdots+\left|x_{i-1}\right|\right)} \\
& \cdot\left[\alpha^{k+k^{\prime}}\left(x_{1}\right), \ldots, \alpha^{k+k^{\prime}}\left(x_{i-1}\right),\right. \\
& \left.\quad\left[D, D^{\prime}\right]\left(x_{i}\right), \alpha^{k+k^{\prime}}\left(x_{i+1}\right), \ldots, \alpha^{k+k^{\prime}}\left(x_{n}\right)\right],
\end{aligned}
$$

which yields that $\left[D, D^{\prime}\right] \in \operatorname{Der}_{\alpha^{k+k^{\prime}}}(\mathfrak{g})$.

Proposition 13. The pair $(\operatorname{Der}(\mathfrak{g}),[\cdot, \cdot])$, where the bracket is the usual commutator, defines a Lie superalgebra and $\operatorname{Inn}(\mathfrak{g})$ constitutes an ideal of it.

Proof. (Der $(\mathfrak{g}),[\cdot, \cdot])$ is a Lie superalgebra by using Lemma 12. We show that $\operatorname{Inn}(\mathfrak{g})$ is an ideal. Let $\operatorname{ad}_{k-1}(\mathscr{X})(y)=$ $\left[x_{1}, \ldots, x_{n-1}, \alpha^{k-1}(y)\right]$ be an inner $\alpha^{k}$-derivation on $\mathfrak{g}$ and 
$D \in \operatorname{Der}_{\alpha^{k^{\prime}}}(\mathfrak{g})$ for $k \geq 1$ and $k^{\prime} \geq 0$. Then $\left[D, \operatorname{ad}_{k-1}(\mathscr{X})\right] \epsilon$ $\operatorname{Der}_{\alpha^{k+k^{\prime}}}(\mathfrak{g})$ and for any $y \in \mathfrak{g}$

$$
\begin{aligned}
& {\left[D, \operatorname{ad}_{k-1}(\mathscr{X})\right](y)} \\
& =D\left[x_{1}, \ldots, x_{n-1}, \alpha^{k-1}(y)\right] \\
& -(-1)^{|D|\left(\left|x_{1}\right|+\cdots+\left|x_{n-1}\right|\right)}\left[x_{1}, \ldots, x_{n-1}, \alpha^{k-1}(D(y))\right] \\
& =D\left[\alpha^{k}\left(x_{1}\right), \ldots, \alpha^{k}\left(x_{n-1}\right), \alpha^{k-1}(y)\right]-(-1)^{|D|\left(\left|x_{1}\right|+\cdots+\left|x_{n-1}\right|\right)} \\
& \cdot\left[\alpha^{k+k^{\prime}}\left(x_{1}\right), \ldots, \alpha^{k+k^{\prime}}\left(x_{n-1}\right), \alpha^{k-1}(D(y))\right] \\
& =\sum_{i \leq n-1}(-1)^{|D|\left(\left|x_{1}\right|+\cdots+\left|x_{i-1}\right|\right)} \\
& \times\left[\alpha^{k+k^{\prime}}\left(x_{1}\right), \ldots, D\left(\alpha^{k}\left(x_{i}\right)\right), \ldots,\right. \\
& \left.\alpha^{k+k^{\prime}}\left(x_{n-1}\right), \alpha^{k+k^{\prime}-1}(y)\right] \\
& =\sum_{i \leq n-1}(-1)^{|D|\left(\left|x_{1}\right|+\cdots+\left|x_{i-1}\right|\right)} \\
& \times\left[x_{1}, \ldots, D\left(x_{i}\right), \ldots, x_{n-1}, \alpha^{k+k^{\prime}-1}(y)\right] \\
& =\sum_{i \leq n-1}(-1)^{|D|\left(\left|x_{1}\right|+\cdots+\left|x_{i-1}\right|\right)} \operatorname{ad}_{k+k^{\prime}-1} \\
& \times\left(x_{1} \wedge \cdots \wedge D\left(x_{i}\right) \wedge \cdots \wedge x_{n-1}\right)(y) .
\end{aligned}
$$

Therefore, $\left[D, \operatorname{ad}_{k-1}(\mathscr{X})\right] \in \operatorname{Inn}_{\alpha^{k+k^{\prime}-1}}(\mathfrak{g})$.

\section{Deformations of $n$-Ary Multiplicative Hom-Nambu-Lie Superalgebras}

Definition 14 (see [33]). For $m \geq 1$, we call $m$-coboundary operator of the $n$-ary multiplicative Hom-Nambu-Lie superalgebra $(\mathfrak{g},[\cdot, \ldots, \cdot], \alpha)$ the even linear map $\delta^{m}: C^{m}(\mathfrak{g}, V) \rightarrow$ $C^{m+1}(\mathfrak{g}, V)$ by

$$
\begin{aligned}
& \left(\delta^{m} f\right)\left(\mathscr{X}_{1}, \ldots, \mathscr{X}_{m}, \mathscr{X}_{m+1}, z\right) \\
& =\sum_{i<j}(-1)^{i}(-1)^{\left|\mathscr{X}_{i}\right|\left(\left|\mathscr{X}_{i+1}\right|+\cdots+\left|\mathscr{X}_{j-1}\right|\right)} \\
& \quad \times f\left(\alpha\left(\mathscr{X}_{1}\right), \ldots, \widehat{\alpha\left(\mathscr{X}_{i}\right)}, \ldots,\right. \\
& +\sum_{i=1}^{m+1}(-1)^{i}(-1)^{\left|\mathscr{X}_{i}\right|\left(\left|\mathscr{X}_{i+1}\right|+\cdots+\left|\mathscr{X}_{m+1}\right|\right)} \\
& \left.\left.\quad \times \mathscr{X}_{i}, \mathscr{X}_{j}\right]_{\alpha}, \ldots, \alpha\left(\mathscr{X}_{m+1}\right), \alpha(z)\right) \\
& +\sum_{i=1}^{m+1}(-1)^{i+1}(-1)^{\left|\mathscr{X}_{i}\right|\left(|f|+\left|\mathscr{X}_{1}\right|+\cdots+\left|\mathscr{X}_{i-1}\right|\right)} \alpha^{m}\left(\mathscr{X}_{i}\right)
\end{aligned}
$$

$$
\begin{aligned}
& \cdot f\left(\mathscr{X}_{1}, \ldots, \widehat{\mathscr{X}}_{i}, \ldots, \mathscr{X}_{m+1}, z\right) \\
& +(-1)^{m}\left(f\left(\mathscr{X}_{1}, \ldots, \mathscr{X}_{m},\right) \cdot \mathscr{X}_{m+1}\right) \cdot{ }_{\alpha} \alpha^{m}(z),
\end{aligned}
$$

where $\mathscr{X}_{i}=\mathscr{X}_{i}^{1} \wedge \cdots \wedge \mathscr{X}_{i}^{n-1} \in \mathfrak{g}^{\wedge^{n-1}}, i=1, \ldots, m+1, z \in \mathfrak{g}$, and the last term is defined by

$$
\begin{gathered}
\left(f\left(\mathscr{X}_{1}, \ldots, \mathscr{X}_{m},\right) \cdot \mathscr{X}_{m+1}\right) \cdot{ }_{\alpha} \alpha^{m}(z) \\
=\sum_{i=1}^{n-1}(-1)^{\left(|f|+\left|X_{1}\right|+\cdots+\left|X_{m}\right|\right)\left(\left|X_{m+1}^{1}\right|+\cdots+\left|X_{m+1}^{i-1}\right|\right)} \\
\cdot\left[\alpha^{m}\left(\mathscr{X}_{m+1}^{1}\right), \ldots, f\left(\mathscr{X}_{1}, \ldots, \mathscr{X}_{m}, \mathscr{X}_{m+1}^{i}\right), \ldots,\right. \\
\left.\alpha^{m}\left(\mathscr{X}_{m+1}^{n-1}\right), \alpha^{m}(z)\right] .
\end{gathered}
$$

Theorem 15 (see [33]). Let $f \in C^{m}(\mathfrak{g}, V)$ be an m-cochain. Then $\delta^{m+1} \circ \delta^{m}(f)=0$.

In [33], it also points out that the map $f \in C^{m}(\mathfrak{g}, V)$ is called an $m$-supercocycle if $\delta^{m} f=0$. We denote by $Z^{m}(\mathfrak{g}, V)$ the graded subspace spanned by $m$-supercocycles. Since $\delta^{m+1} \circ \delta^{m}(f)=0$ for all $f \in C^{m}(\mathfrak{g}, V), \delta^{m-1} C^{m-1}(\mathfrak{g}, V)$ is a graded subspace of $Z^{m}(\mathfrak{g}, V)$. Therefore, we can define a graded cohomology space $H^{m}(\mathfrak{g}, V)$ of $\mathfrak{g}$ as the graded factor space $Z^{m}(\mathfrak{g}, V) / \delta^{m-1} C^{m-1}(\mathfrak{g}, V)$.

We next will discuss the deformation of $n$-ary multiplicative Hom-Nambu-Lie superalgebras. Let $\mathbb{K}[[t]]$ denote the power series ring in one variable $t$ with coefficients in $\mathbb{K}$ and let $\mathfrak{g}[[t]]$ be the set of formal series whose coefficients are elements of the vector space $\mathfrak{g}$.

Definition 16. Let $(\mathfrak{g},[\cdot, \ldots, \cdot], \alpha)$ be an $n$-ary multiplicative Hom-Nambu-Lie superalgebra over $\mathbb{K}$. A deformation of $(\mathfrak{g},[\cdot, \ldots, \cdot], \alpha)$ is given by $\mathbb{K}[[t]]-n$-linear map

$$
f_{t}=\sum_{p \geq 0} f_{p} t^{p}: \mathfrak{g}[[t]] \times \cdots \times \mathfrak{g}[[t]] \longrightarrow \mathfrak{g}[[t]]
$$

such that $\left(\mathfrak{g}[[t]], f_{t}, \alpha\right)$ is also an $n$-ary multiplicative HomNambu-Lie superalgebra. We call $f_{1}$ the infinitesimal deformation of $(\mathfrak{g},[\cdot, \ldots, \cdot], \alpha)$.

Since $\left(\mathfrak{g}[[t]], f_{t}, \alpha\right)$ is an $n$-ary multiplicative HomNambu-Lie superalgebra, $f_{t}$ satisfies

$$
\begin{gathered}
\alpha \circ f_{t}\left(x_{1}, \ldots, x_{n}\right)=f_{t}\left(\alpha\left(x_{1}\right), \ldots, \alpha\left(x_{n}\right)\right), \\
\left|f_{t}\left(x_{1}, \ldots, x_{n}\right)\right|=\left|x_{1}\right|+\cdots+\left|x_{n}\right|, \\
f_{t}\left(\alpha\left(x_{1}\right), \ldots, \alpha\left(x_{n-1}\right), f_{t}\left(y_{1}, \ldots, y_{n}\right)\right) \\
=\sum_{i=1}^{n}(-1)^{\left(\left|x_{1}\right|+\cdots+\left|x_{n-1}\right|\right)\left(\left|y_{1}\right|+\cdots+\left|y_{i-1}\right|\right)} \\
\cdot f_{t}\left(\alpha\left(y_{1}\right), \ldots, \alpha\left(y_{i-1}\right), f_{t}\left(x_{1}, \ldots, x_{n-1}, y_{i}\right),\right. \\
\left.\alpha\left(y_{i+1}\right), \ldots, \alpha\left(y_{n}\right)\right) .
\end{gathered}
$$


Equations (27)-(29) are, respectively, equivalent to

$$
\begin{gathered}
\alpha \circ f_{p}\left(x_{1}, \ldots, x_{n}\right)=f_{p}\left(\alpha\left(x_{1}\right), \ldots, \alpha\left(x_{n}\right)\right), \quad\left(27^{\prime}\right) \\
\left|f_{p}\left(x_{1}, \ldots, x_{n}\right)\right|=\left|x_{1}\right|+\cdots+\left|x_{n}\right|, \quad\left(28^{\prime}\right) \\
\sum_{p+q=l} f_{p}\left(\alpha\left(x_{1}\right), \ldots, \alpha\left(x_{n-1}\right), f_{q}\left(y_{1}, \ldots, y_{n}\right)\right) \\
=\sum_{i=1}^{n}(-1)^{\left(\left|x_{1}\right|+\cdots+\left|x_{n-1}\right|\right)\left(\left|y_{1}\right|+\cdots+\left|y_{i-1}\right|\right)} \\
\cdot\left(\sum _ { p + q = l } f _ { p } \left(\alpha\left(y_{1}\right), \ldots, \alpha\left(y_{i-1}\right),\right.\right. \\
\left.\left.f_{q}\left(x_{1}, \ldots, x_{n-1}, y_{i}\right), \alpha\left(y_{i+1}\right), \ldots, \alpha\left(y_{n}\right)\right)\right) .
\end{gathered}
$$

We call these the deformation equations for an $n$-ary multiplicative Hom-Nambu-Lie superalgebra.

Equations $\left(27^{\prime}\right)$ and $\left(28^{\prime}\right)$ show that $f_{p} \in C^{1}(\mathfrak{g}, \mathfrak{g})_{\overline{0}}$. In $\left(29^{\prime}\right)$, set $l=1$, and then

$$
\begin{aligned}
& {\left[\alpha\left(x_{1}\right), \ldots, \alpha\left(x_{n-1}\right), f_{1}\left(y_{1}, \ldots, y_{n}\right)\right]} \\
& +f_{1}\left(\alpha\left(x_{1}\right), \ldots, \alpha\left(x_{n-1}\right),\left[y_{1}, \ldots, y_{n}\right]\right) \\
& -\sum_{i=1}^{n}(-1)^{\left(\left|x_{1}\right|+\cdots+\left|x_{n-1}\right|\right)\left(\left|y_{1}\right|+\cdots+\left|y_{i-1}\right|\right)} \\
& \quad \times\left(\left[\alpha\left(y_{1}\right), \ldots, \alpha\left(y_{i-1}\right), f_{1}\left(x_{1}, \ldots, x_{n-1}, y_{i}\right),\right.\right. \\
& \left.\quad \alpha\left(y_{i+1}\right), \ldots, \alpha\left(y_{n}\right)\right] \\
& +f_{1}\left(\alpha\left(y_{1}\right), \ldots, \alpha\left(y_{i-1}\right),\right. \\
& \left.\left.\left[x_{1}, \ldots, x_{n-1}, y_{i}\right], \alpha\left(y_{i+1}\right), \ldots, \alpha\left(y_{n}\right)\right)\right)
\end{aligned}
$$$$
=0 \text {; }
$$

that is, $\delta^{1} f_{1}\left(x_{1}, \ldots, x_{n-1},\left[y_{1}, \ldots, y_{n-1}, y_{n}\right]\right)=0$. Hence the infinitesimal deformation $f_{1} \in Z^{1}(\mathfrak{g}, \mathfrak{g})_{\overline{0}}$.

Definition 17. Two deformations $f_{t}$ and $f_{t^{\prime}}$ of the $n$-ary multiplicative Hom-Nambu-Lie superalgebra $(\mathfrak{g},[\cdot, \cdots, \cdot], \alpha)$ are said to be equivalent, if there exists an isomorphism of $n$-ary multiplicative Hom-Nambu-Lie superalgebras $\Phi_{t}$ : $\left(\mathfrak{g}, f_{t}, \alpha\right) \rightarrow\left(\mathfrak{g}, f_{t}^{\prime}, \alpha\right)$, where $\Phi_{t}=\sum_{i \geq 0} \varphi_{i} t^{i}, \varphi_{i}: \mathfrak{g} \rightarrow \mathfrak{g}$ is a linear map such that

$$
\begin{gathered}
\varphi_{0}=\mathrm{id}_{\mathfrak{g}} ; \quad \varphi_{i} \circ \alpha=\alpha \circ \varphi_{i} ; \\
\Phi_{t} \circ f_{t}\left(x_{1}, \ldots, x_{n}\right)=f_{t}^{\prime}\left(\Phi_{t}\left(x_{1}\right), \ldots, \Phi_{t}\left(x_{n}\right)\right)
\end{gathered}
$$

and is denoted by $f_{t} \sim f_{t^{\prime}}$. When $f_{1}=f_{2}=\cdots=0, f_{t}=f_{0}$ is called the null deformation; if $f_{t} \sim f_{0}$, then $f_{t}$ is called the trivial deformation.
Theorem 18. Let $f_{t}$ and $f_{t^{\prime}}$ be two equivalent deformations of the n-ary multiplicative Hom-Nambu-Lie superalgebra $(\mathfrak{g},[\cdot, \ldots, \cdot], \alpha)$. Then the infinitesimal deformations $f_{1}$ and $f_{1}^{\prime}$ belong to the same cohomology class in the cohomology group $H^{2}(\mathfrak{g}, \mathfrak{g})$.

Proof. Put $B^{2}(\mathfrak{g}, \mathfrak{g}):=\delta^{1} C^{1}(\mathfrak{g}, \mathfrak{g})$. It is enough to prove that $f_{1}-f_{1}^{\prime} \in B^{2}(\mathfrak{g}, \mathfrak{g})$. Let $\Phi_{t}:\left(\mathfrak{g}, f_{t}, \alpha\right) \rightarrow\left(\mathfrak{g}, f_{t}^{\prime}, \alpha\right)$ be an isomorphism of $n$-ary multiplicative Hom-Nambu-Lie superalgebras. Then $\varphi_{1} \in C^{1}(\mathfrak{g}, \mathfrak{g})_{\overline{0}}$ and

$$
\begin{aligned}
\sum_{i \geq 0} \varphi_{i}\left(\sum_{j \geq 0} f_{j}\left(x_{1}, \ldots, x_{n}\right)\right) t^{i+j} \\
\quad=\sum_{i \geq 0} f_{i}^{\prime}\left(\sum_{j_{1} \geq 0} \varphi_{j_{1}}\left(x_{1}\right), \ldots, \sum_{j_{n} \geq 0} \varphi_{j_{n}}\left(x_{n}\right)\right) t^{i+j_{1}+\cdots+j_{n}},
\end{aligned}
$$

and comparing with the coefficients of $t^{1}$ for two sides of the above equation, we obtain

$$
\begin{aligned}
f_{1}( & \left.x_{1}, \ldots, x_{n}\right)+\varphi_{1}\left[x_{1}, x_{2}, \ldots, x_{n}\right] \\
= & {\left[\varphi_{1}\left(x_{1}\right), x_{2}, \ldots, x_{n}\right]+\left[x_{1}, \varphi_{1}\left(x_{2}\right), x_{3}, \ldots, x_{n}\right] } \\
& +\cdots+\left[x_{1}, \ldots, x_{n-1}, \varphi_{1}\left(x_{n}\right)\right]+f_{1}^{\prime}\left(x_{1}, \ldots, x_{n}\right) .
\end{aligned}
$$

Furthermore, one gets

$$
\begin{aligned}
f_{1}( & \left.x_{1}, \ldots, x_{n}\right)-f_{1}^{\prime}\left(x_{1}, \ldots, x_{n}\right) \\
= & -\varphi_{1}\left[x_{1}, x_{2}, \ldots, x_{n}\right]+\left[\varphi_{1}\left(x_{1}\right), x_{2}, \ldots, x_{n}\right] \\
& +\left[x_{1}, \varphi_{1}\left(x_{2}\right), x_{3}, \ldots, x_{n}\right]+\cdots+\left[x_{1}, \ldots, x_{n-1}, \varphi_{1}\left(x_{n}\right)\right] \\
= & -\varphi_{1}\left[x_{1}, \ldots, x_{n}\right] \\
& +\sum_{i=1}^{n}(-1)^{n-i}(-1)^{\left|x_{i}\right|\left(\left|x_{i+1}\right|+\cdots+\left|x_{n}\right|\right)} \\
& \quad \times\left[x_{1}, \ldots, \widehat{x}_{i}, \ldots, x_{n}, \varphi_{1}\left(x_{i}\right)\right] \\
= & \delta^{1} \varphi_{1}\left(x_{1}, \ldots, x_{n}\right) .
\end{aligned}
$$

Therefore, $f_{1}-f_{1}^{\prime}=\delta^{1} \varphi_{1} \in \delta^{1} C^{1}(\mathfrak{g}, \mathfrak{g})_{\overline{0}}$; that is, $f_{1}-f_{1}^{\prime} \in$ $B^{2}(\mathfrak{g}, \mathfrak{g})$.

An $n$-ary multiplicative Hom-Nambu-Lie superalgebra $(\mathfrak{g},[\cdot, \cdots, \cdot], \alpha)$ is analytically rigid if every deformation $f_{t}$ is equivalent to the null deformation $f_{0}$. We have a fundamental theorem.

Theorem 19. If $(\mathfrak{g},[\cdot, \ldots, \cdot], \alpha)$ is an $n$-ary multiplicative Hom-Nambu-Lie superalgebra with $H^{2}(\mathfrak{g}, \mathfrak{g})=0$, then $(\mathfrak{g},[\cdot, \ldots, \cdot], \alpha)$ is analytically rigid.

Proof. Let $f_{t}$ be a deformation of the $n$-ary multiplicative Hom-Nambu-Lie superalgebra $(\mathfrak{g},[\cdot, \ldots, \cdot], \alpha)$ with $f_{t}=f_{0}+$ 
$f_{r} t^{r}+f_{r+1} t^{r+1}+\cdots ;$ that is; $f_{1}=f_{2}=\cdots=f_{r-1}=0$. Then setting $l=r$ in $\left(29^{\prime}\right)$, we have

$$
\begin{gathered}
f_{r}\left(\alpha\left(x_{1}\right), \ldots, \alpha\left(x_{n-1}\right),\left[y_{1}, \ldots, y_{n}\right]\right) \\
+\left[\alpha\left(x_{1}\right), \ldots, \alpha\left(x_{n-1}\right), f_{r}\left(y_{1}, \ldots, y_{n}\right)\right] \\
-\sum_{i=1}^{n}(-1)^{\left(\left|x_{1}\right|+\cdots+\left|x_{n-1}\right|\right)\left(\left|y_{1}\right|+\cdots+\left|y_{i-1}\right|\right)} \\
\cdot\left(\left[\alpha\left(y_{1}\right), \ldots, \alpha\left(y_{i-1}\right), f_{r}\left(x_{1}, \ldots, x_{n-1}, y_{i}\right),\right.\right. \\
\left.\alpha\left(y_{i+1}\right), \ldots, \alpha\left(y_{n}\right)\right] \\
+f_{r}\left(\alpha\left(y_{1}\right), \ldots, \alpha\left(y_{i-1}\right),\left[x_{1}, \ldots, x_{n-1}, y_{i}\right],\right. \\
\left.\left.\alpha\left(y_{i+1}\right), \ldots, \alpha\left(y_{n}\right)\right)\right)=0,
\end{gathered}
$$

that is, $\delta^{2} f_{r}\left(x_{1}, \ldots, x_{n-1},\left[y_{1}, \ldots, y_{n}\right]\right)=0, \delta^{2}\left(f_{r}\right)=0$; that is, $f_{r} \in Z^{2}(\mathfrak{g}, \mathfrak{g})_{0}$. By our assumption $H^{2}(\mathfrak{g}, \mathfrak{g})=0$, one gets $f_{r} \in B^{2}(\mathfrak{g}, \mathfrak{g})_{\overline{0}}$, and thus we can find $h_{r} \in C^{1}(\mathfrak{g}, \mathfrak{g})_{0}$ such that $f_{r}=\delta^{1} h_{r}$. Putting $\Phi_{t}=\mathrm{id}_{\mathfrak{g}}-h_{r} t^{r}$, then

$$
\begin{aligned}
\Phi_{t} \circ & \left(\mathrm{id}_{\mathfrak{g}}+h_{r} t^{r}+h_{r}^{2} t^{2 r}+h_{r}^{3} t^{3 r}+\cdots\right) \\
= & \left(\mathrm{id}_{\mathfrak{g}}-h_{r} t^{r}\right) \circ\left(\mathrm{id}_{\mathfrak{g}}+h_{r} t^{r}+h_{r}^{2} t^{2 r}+h_{r}^{3} t^{3 r}+\cdots\right) \\
= & \left(\mathrm{id}_{\mathfrak{g}}+h_{r} t^{r}+h_{r}^{2} t^{2 r}+h_{r}^{3} t^{3 r}+\cdots\right) \\
& -\left(h_{r} t^{r}+h_{r}^{2} t^{2 r}+h_{r}^{3} t^{3 r}+\cdots\right) \\
= & \mathrm{id}_{\mathfrak{g}}
\end{aligned}
$$

and moreover, $\left(\mathrm{id}_{\mathfrak{g}}+h_{r} t^{r}+h_{r}^{2} t^{2 r}+h_{r}^{3} t^{3 r}+\cdots\right) \circ \Phi_{t}=\mathrm{id}_{\mathfrak{g}}$. Hence $\Phi_{t}: \mathfrak{g} \rightarrow \mathfrak{g}$ is a linear isomorphism and $\Phi_{t} \circ \alpha=$ $\alpha \circ \Phi_{t}$. Set $f_{t}^{\prime}\left(x_{1}, \ldots, x_{n}\right)=\Phi_{t}^{-1} f_{t}\left(\Phi_{t}\left(x_{1}\right), \ldots, \Phi_{t}\left(x_{n}\right)\right)$, and then $f_{t}^{\prime}$ is also a deformation of $(\mathfrak{g},[\cdot, \ldots, \cdot], \alpha)$ and $f_{t} \sim f_{t}^{\prime}$. Note that $\Phi_{t} f_{t}^{\prime}\left(x_{1}, \ldots, x_{n}\right)=f_{t}\left(\Phi_{t}\left(x_{1}\right), \ldots, \Phi_{t}\left(x_{n}\right)\right)$. Let $f_{t}^{\prime}=$ $\sum_{i \geq 0} f_{i}^{\prime} t^{i}$. Then

$$
\begin{aligned}
\left(\mathrm{id}_{\mathfrak{g}}\right. & \left.h_{r} t^{r}\right) \sum_{i \geq 0} f_{i}^{\prime}\left(x_{1}, \ldots, x_{n}\right) t^{i} \\
& =\left(f_{0}+\sum_{i \geq r} f_{i} t^{i}\right)\left(x_{1}-h_{r}\left(x_{1}\right) t^{r}, \ldots, x_{n}-h_{r}\left(x_{n}\right) t^{r}\right) .
\end{aligned}
$$

So

$$
\begin{aligned}
& \sum_{i \geq 0} f_{i}^{\prime}\left(x_{1}, \ldots, x_{n}\right) t^{i}-\sum_{i \geq 0} h_{r} \circ f_{i}^{\prime}\left(x_{1}, \ldots, x_{n}\right) t^{i+r} \\
& =f_{0}\left(x_{1}, \ldots, x_{n}\right)-\sum_{i=1}^{n} f_{0}\left(x_{1}, \ldots, h_{r}\left(x_{i}\right), \ldots, x_{n}\right) t^{r} \\
& \quad+\sum_{1 \leq i<j \leq n} f_{0}\left(x_{1}, \ldots, h_{r}\left(x_{i}\right), \ldots, h_{r}\left(x_{j}\right), \ldots, x_{n}\right) t^{2 r}
\end{aligned}
$$

$$
\begin{aligned}
& -\sum_{1 \leq i<j<k \leq n} f_{0}\left(x_{1}, \ldots, h_{r}\left(x_{i}\right), \ldots, h_{r}\left(x_{j}\right), \ldots,\right. \\
& \left.h_{r}\left(x_{k}\right), \ldots, x_{n}\right) t^{3 r}+\ldots \\
& +(-1)^{n} f_{0}\left(h_{r}\left(x_{1}\right), h_{r}\left(x_{2}\right), \ldots, h_{r}\left(x_{n}\right)\right) t^{n r} \\
& +\sum_{i \geq r} f_{i}\left(x_{1}, \ldots, x_{n}\right) t^{i} \\
& -\sum_{i \geq r} \sum_{j=1}^{n} f_{i}\left(x_{1}, \ldots, h_{r}\left(x_{j}\right), \ldots, x_{n}\right) t^{i+r} \\
& +\sum_{i \geq r} \sum_{1 \leq j \leq k \leq n} f_{i}\left(x_{1}, \ldots, h_{r}\left(x_{j}\right), \ldots, h_{r}\left(x_{k}\right), \ldots\right. \\
& \left.x_{n}\right) t^{i+2 r}+\ldots .
\end{aligned}
$$

By the above equation, one gets

$$
\begin{gathered}
f_{0}^{\prime}\left(x_{1}, \ldots, x_{n}\right)=f_{0}\left(x_{1}, \ldots, x_{n}\right)=\left[x_{1}, \ldots, x_{n}\right] ; \\
f_{1}^{\prime}\left(x_{1}, \ldots, x_{n}\right)=\cdots=f_{r-1}^{\prime}\left(x_{1}, \ldots, x_{n}\right)=0 ; \\
f_{r}^{\prime}\left(x_{1}, \ldots, x_{n}\right)-h_{r}\left[x_{1}, \ldots, x_{n}\right] \\
=-\sum_{i=1}^{n}\left[x_{1}, \ldots, h_{r}\left(x_{i}\right), \ldots, x_{n}\right]+f_{r}\left(x_{1}, \ldots, x_{n}\right) .
\end{gathered}
$$

Furthermore, we have

$$
f_{r}^{\prime}\left(x_{1}, \ldots, x_{n}\right)=-\delta^{1} h_{r}\left(x_{1}, \ldots, x_{n}\right)+f_{r}\left(x_{1}, \ldots, x_{n}\right)=0,
$$

and hence, $f_{t}^{\prime}=f_{0}+\sum_{i \geq r+1} f_{i}^{\prime} t^{i}$. By induction, one can prove $f_{t} \sim f_{0}$; that is, $(\mathfrak{g},[\cdot, \ldots, \cdot], \alpha)$ is analytically rigid.

\section{Conflict of Interests}

The authors declare that there is no conflict of interests regarding the publication of this paper.

\section{Acknowledgments}

This research is supported by NNSF of China (no. 11171055), NSF of Jilin (no. 201115006), Scientific Research Fund of Heilongjiang Provincial Education Department (no. 12541900), Scientific Research Foundation for Returned Scholars Ministry of Education of China, and the Fundamental Research Funds for the Central Universities (no. 12SSXT139).

\section{References}

[1] V. T. Filippov, " $n$-Lie algebras," Akademiya Nauk SSSR. Sibirskoe Otdelenie. Sibirskiu Matematicheskiu Zhurnal, vol. 26, no. 6, pp. 126-140, 1985 (Russian).

[2] Y. Nambu, "Generalized Hamiltonian dynamics," Physical Review D, vol. 7, pp. 2405-2412, 1973. 
[3] J. Bagger and N. Lambert, "Gauge symmetry and supersymmetry of multiple M2-branes," Physical Review D, vol. 77, no. 6, Article ID 065008, 2008.

[4] J. Bagger and N. Lambert, "Comments on multiple M2-branes," Journal of High Energy Physics, vol. 105, no. 2, p. 15, 2008.

[5] A. Gustavsson, "Algebraic structures on parallel M2 branes," Nuclear Physics B, vol. 811, no. 1-2, pp. 66-76, 2009.

[6] G. Papadopoulos, "M2-branes, 3-Lie algebras and Plücker relations," Journal of High Energy Physics, no. 5, p. 9, 2008.

[7] Yu. L. Daletskii and V. A. Kushnirevich, "Inclusion of the Nambu-Takhtajan algebra in the structure of formal differential geometry," Dopovidi Natsional'noyi Akademiyi Nauk Ukrayini, no. 4, pp. 12-18, 1996.

[8] N. Cantarini and V. G. Kac, "Classification of simple linearly compact $n$-Lie superalgebras," Communications in Mathematical Physics, vol. 298, no. 3, pp. 833-853, 2010.

[9] F. Ammar, Z. Ejbehi, and A. Makhlouf, "Cohomology and deformations of Hom-algebras," Journal of Lie Theory, vol. 21, no. 4, pp. 813-836, 2011.

[10] F. Ammar, S. Mabrouk, and A. Makhlouf, "Representations and cohomology of $n$-ary multiplicative Hom-Nambu-Lie algebras," Journal of Geometry and Physics, vol. 61, no. 10, pp. 1898-1913, 2011.

[11] F. Ammar and A. Makhlouf, "Hom-Lie superalgebras and HomLie admissible superalgebras," Journal of Algebra, vol. 324, no. 7, pp. 1513-1528, 2010.

[12] R. P. Bai and Y. Li, "Extensions of $n$-Hom Lie algebras," Frontiers of Mathematics in China, 2014.

[13] S. Benayadi and A. Makhlouf, "Hom-Lie algebras with symmetric invariant nondegenerate bilinear forms," Journal of Geometry and Physics, vol. 76, pp. 38-60, 2014.

[14] J. T. Hartwig, D. Larsson, and S. D. Silvestrov, "Deformations of Lie algebras using $\sigma$-derivations," Journal of Algebra, vol. 295, no. 2, pp. 314-361, 2006.

[15] D. Larsson and S. D. Silvestrov, "Quasi-hom-Lie algebras, central extensions and 2-cocycle-like identities," Journal of Algebra, vol. 288, no. 2, pp. 321-344, 2005.

[16] A. Makhlouf and S. Silvestrov, "Notes on 1-parameter formal deformations of Hom-associative and Hom-Lie algebras," Forum Mathematicum, vol. 22, no. 4, pp. 715-739, 2010.

[17] A. Makhlouf and S. Silvestrov, "Hom-algebras and Homcoalgebras," Journal of Algebra and its Applications, vol. 9, no. 4, pp. 553-589, 2010.

[18] Y. Sheng, "Representations of hom-Lie algebras," Algebras and Representation Theory, vol. 15, no. 6, pp. 1081-1098, 2012.

[19] D. Yau, "The Hom-Yang-Baxter equation, Hom-Lie algebras, and quasi-triangular bialgebras," Journal of Physics A. Mathematical and Theoretical, vol. 42, no. 16, Article ID 165202, 2009.

[20] D. Yau, "Hom-algebras and homology," Journal of Lie Theory, vol. 19, no. 2, pp. 409-421, 2009.

[21] H. Ataguema, A. Makhlouf, and S. Silvestrov, "Generalization of $n$-ary Nambu algebras and beyond," Journal of Mathematical Physics, vol. 50, no. 8, 2009.

[22] M. Gerstenhaber, "The cohomology structure of an associative ring," Annals of Mathematics, vol. 78, pp. 267-288, 1963.

[23] M. Gerstenhaber, "On the deformation of rings and algebras," Annals of Mathematics, vol. 79, pp. 59-103, 1964.

[24] M. Gerstenhaber, "On the deformation of rings and algebras. II," Annals of Mathematics, vol. 84, pp. 1-19, 1966.
[25] M. Gerstenhaber, "On the deformation of rings and algebras. III," Annals of Mathematics, vol. 88, pp. 1-34, 1968.

[26] M. Gerstenhaber, "On the deformation of rings and algebras. IV,' Annals of Mathematics, vol. 99, pp. 257-276, 1974.

[27] M. Gerstenhaber and S. D. Schack, "Algebras, bialgebras, quantum groups, and algebraic deformations," in Deformation Theory and Quantum Groups with Applications to Mathematical Physics, M. Gerstenhaber and J. Stasheff, Eds., vol. 134 of Contemporary Mathematics, pp. 51-92, American Mathematical Society, Providence, RI, USA, 1992.

[28] M. Flato, M. Gerstenhaber, and A. A. Voronov, "Cohomology and deformation of Leibniz pairs," Letters in Mathematical Physics, vol. 34, no. 1, pp. 77-90, 1995.

[29] A. Nijenhuis and R. W. Richardson, Jr., "Cohomology and deformations of algebraic structures," Bulletin of the American Mathematical Society, vol. 70, pp. 406-411, 1964.

[30] A. Nijenhuis and R. W. Richardson Jr., "Cohomology and deformations in graded Lie algebras," Bulletin of the American Mathematical Society, vol. 72, pp. 1-29, 1966.

[31] A. Nijenhuis and R. W. Richardson Jr., "Deformations of homomorphisms of Lie groups and Lie algebras," Bulletin of the American Mathematical Society, vol. 73, pp. 175-179, 1967.

[32] F. Ammar and N. Saadaoui, "Cohomology of $n$-ary-NambuLie superalgebras and super $\omega_{\mathrm{o}} 3$-algebra," http://arxiv-web3. library.cornell.edu/abs/1304.5767v1.

[33] B. L. Guan, L. Y. Chen, and Y. Ma, "On the cohomology and extensions of $n$-ary multiplicative Hom-Nambu-Lie superalgebras," http://arxiv.org/abs/1401.0378. 


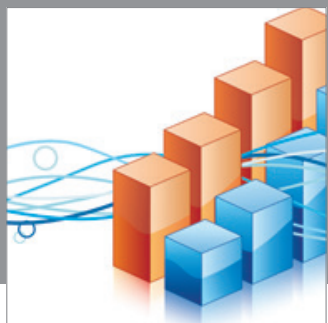

Advances in

Operations Research

mansans

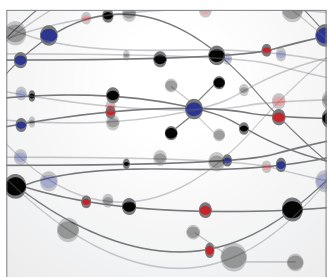

The Scientific World Journal
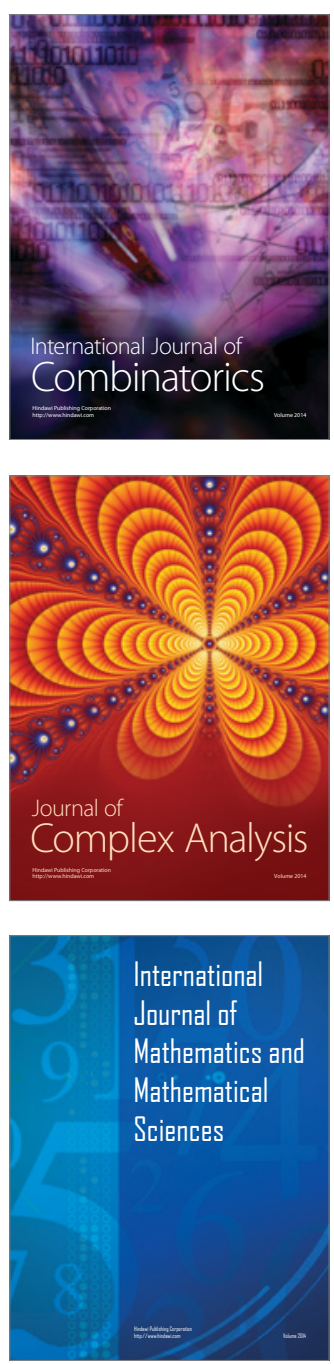
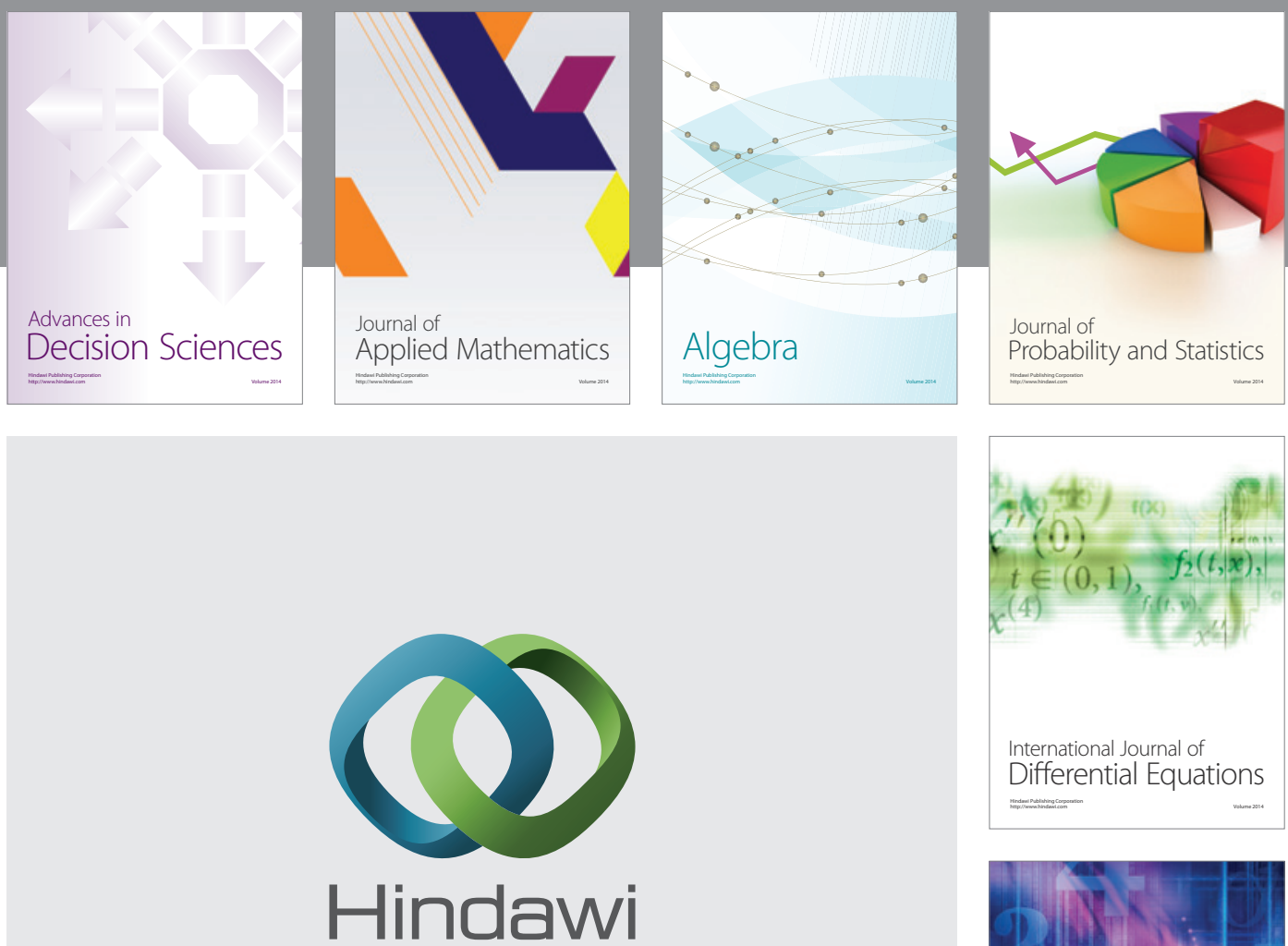

Submit your manuscripts at http://www.hindawi.com
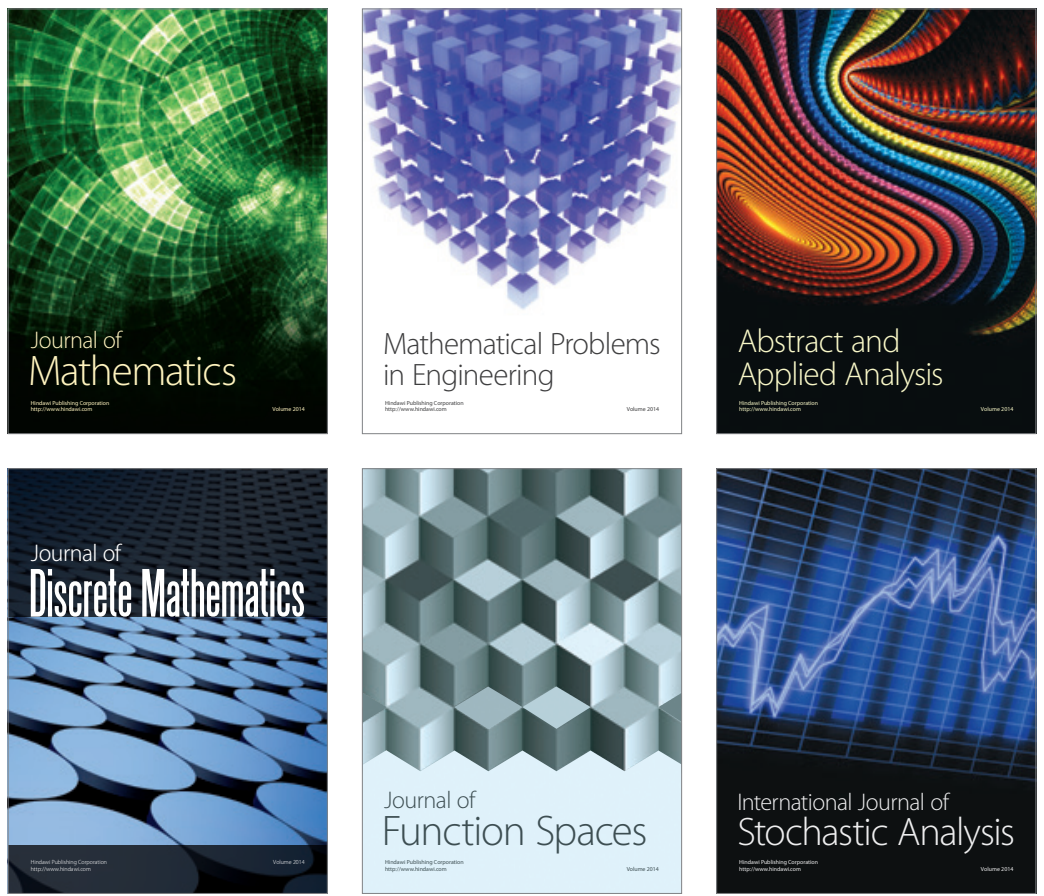

Journal of

Function Spaces

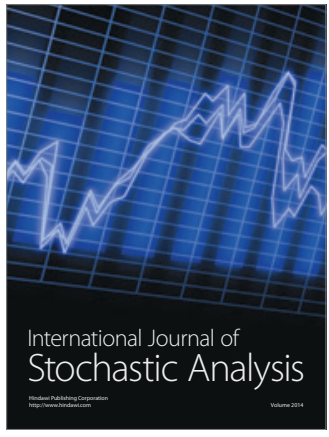

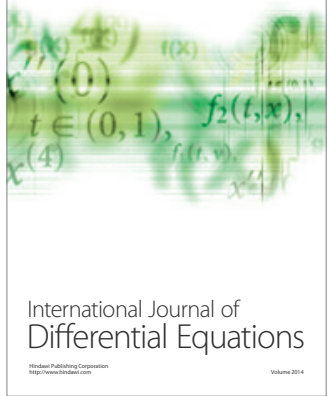
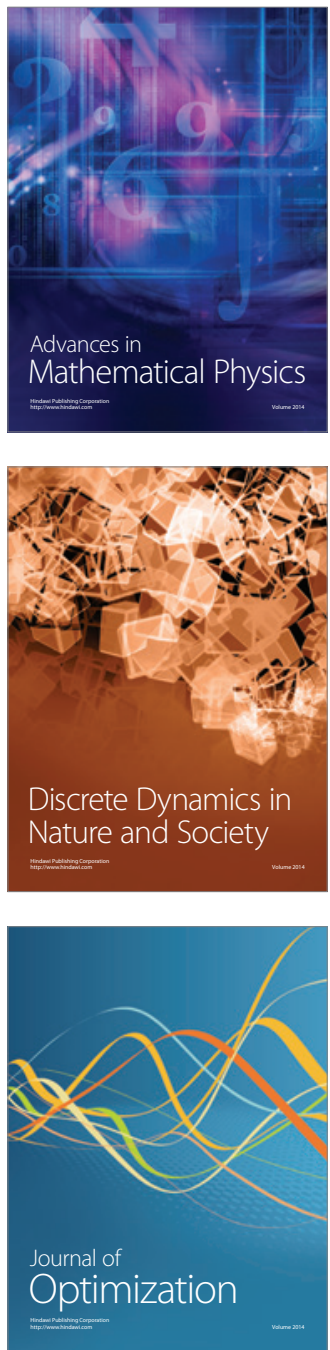